\title{
An analysis of the monaural displacement of sound in space
}

\author{
ROBERT A. BUTLER \\ University of Chicago, Chicago, llinois
}

\begin{abstract}
Twelve listeners, rendered monaural, were tested on their ability to localize two spectrally different stimuli presented $10^{\circ}$ apart in the left hemifield (pretraining session). Both sounds were perceived to be displaced from the midline. Next, listeners were trained to localize one of the sounds (training sessions) and then tested again on both (posttraining sessions). Training temporarily reduced the extent to which listeners displaced the sound from the midline. No transfer of training, however, was observed when listeners localized the other noise band, which differed in its spectral content. It is proposed that no "natural" spectral cues are available for localizing, monaurally, sound originating on or near the midline, but that cues for these positions can be acquired through training. Head-related transfer functions recorded from the pinna and generated by differently centered narrow bands of noise were obtained, along with additional localization data, in an attempt to support the concept of a natural cue for monaurally localizing sound in the horizontal plane. By and large, the results were consonant with this concept.
\end{abstract}

"Since the work of Bloch in 1893, it has been generally recognized without serious contradiction, that the localization of sounds in the horizontal plane is most accurate in the region directly in front or behind the O[bserver]' (Stevens \& Newman, 1936, p. 303). This statement still holds true for binaural localization of complex sounds, but it is precisely in the midline region that accuracy of monaural localization is poorest. The apparent location of all sounds is displaced toward the functioning ear. References to this phenomenon are scattered throughout the literature dating back at least to the turn of the century (see Pierce, 1901, for a review of these early studies). Monaural displacement of sound has not been subjected to an experimental analysis, a fact that, paradoxically, may account for the wide acceptance of one explanation for the frequently observed event, namely, an imbalance in the auditory input. In brief, for the listener to locate accurately a sound originating at the midline, the binaural differences in time and intensity must be absent or minimal. This condition is obviously violated when only one ear is stimulated. Another explanation exists: Given that monaural localization is based on spectral cues provided primarily by the pinna, it is entirely possible that no "natural" cue is available for locating sounds emanating from the vicinity of the midline.

The question immediately arises, What constitutes a natural cue? The underlying assumption of the present study is that it has something to do with the peak in the sound's spectrum as modified by the pinna. Shaw (1974) showed that the pinna differentially filters the spectral con-

This research was supported in part by a Biomedical Research Support Grant (PHS 2 S07-07029). The author thanks Joel Woller for his assistance. The author's address is: Department of Surgery (Otolaryngology), University of Chicago Medical Center, 5841 South Maryland Avenue, Box 412, Chicago, IL 60637. tent of a sound; different frequency segments are variously amplified or attenuated, depending on the azimuthal positions of their sources. When measured at the ear canal entrance, every sound generated in space contains a frequency segment that is amplified as much as or more than other segments. But if a spectral peak is indeed a cue for monaural localization, the overt peak is a poor candidate, because, over the front quadrant of the horizontal plane, the frequency segments centered about 4.0 and $5.0 \mathrm{kHz}$ are amplified more than are segments of higher audio frequencies (Musicant \& Butler, 1984). Hence, I suggest that covert peaks in the spectrum are natural cues for localizing sound monaurally. A spectral cue in this sense is that frequency segment within a given sound that is amplified more when the sound originates from one specific azimuthal position than when it originates from any other position. Hence, that frequency segment is the cue for sound coming from that azimuthal position. The data of Musicant and Butler (1984) lend credence to this idea. They reported a high correlation between the azimuthal positions of the covert peaks generated by $1.0-\mathrm{kHz}$-wide noise bands whose center frequencies differed widely and the apparent locations of these narrow bands in the horizontal plane.

Every frequency segment peaks covertly when the sound is generated somewhere in the horizontal plane, thereby providing a spectral cue for localization. But not all azimuthal positions from which a sound originates will necessarily generate a spectral cue as defined above. If a sound's spectral content does not contain a natural cue for a specific position, then monaural listeners will be unlikely to localize the sound when it originates from that place. Therefore, when sounds originate on or near the midline, monaural listeners perceive them to be displaced toward the functioning ear. For this situation, I propose that some characteristic of the sound's spectrum other than 
covert peaks is utilized as a cue for location, and that this cue must be acquired through training. In this connection, Musicant and Butler (1980) showed that practice in localizing, monaurally, a wider band stimulus $(>3.0 \mathrm{kHz}$ ) enabled listeners to judge more accurately the position of sounds originating in the vicinity of the midline. It can be assumed that, if no natural cue was available for localizing midline-positioned sounds, the listeners learned something about the spectrum, perhaps its pattern, that was highly specific to the frequency content of the stimulus. If this is true, learning to localize, monaurally, a sound that comprises one band of frequencies should not improve performance in locating a sound that comprises a different band. This prediction was tested in Experiment 1. In Experiment 2, an attempt was made to account for the apparent location of the sounds presented in Experiment 1 in terms of covert spectral peaks. Head-related transfer functions (HRTFs), recorded at the entrance of the external ear canal, were obtained for each listener, and the degree of correspondence between covert peaks and location judgments was examined.

\section{EXPERIMENT 1}

\section{The Effect of Practice in Sound Localization}

\section{Method}

Twelve undergraduate students whose absolute auditory thresholds were within $10 \mathrm{~dB}$ of audiometric zero (ANSI, 1969) for frequencies from 0.25 through $8.0 \mathrm{kHz}$ participated. They were rendered monaural by placing an E-A-R insert into the external ear canal of the right ear and then covering that ear with a Mine Safety Appliance earmuff. All tests were conducted in a sound-treated room, $4.9 \times 4.1 \times 2.1 \mathrm{~m}$, whose reverberation time was approximatly $75 \mathrm{msec}$. The listeners were required to call out the number of the loudspeaker from which a train of noise bursts emerged. Nineteen loudspeakers (Braun, Output Compact/C) were positioned $10^{\circ}$ apart in the horizontal plane of the left hemifield. Loudspeaker No. 1 was stationed at $0^{\circ}$ azimuth and No. 19 at $180^{\circ}$. The subjects, tested individually, were given a diagram of the loudspeaker arrangement to aid them in identifying the loudspeaker being activated. They were instructed not to move their heads. A headrest was attached to the back of the chair to promote compliance with this instruction. The experimenter could observe the subject through a window in the wall that separated the test room from that housing the sound-generating equipment.

Prior to the first formal test session, the listeners were requested to locate a white (broadband) noise stimulus presented at a mean sensation level of $30 \mathrm{~dB}$. On prearranged trials, the stimulus level was increased or decreased by $3 \mathrm{~dB}$ re the $30 \mathrm{~dB}$ SL. The purpose of this test was to demonstrate that when a wide range of spectral cues are available, naive monaural listeners still displace sounds away from the midline and toward the functioning ear. Stimulus duration was $30 \mathrm{msec}$, rise-fall time was $10 \mathrm{msec}$, and interstimulus interval was $300 \mathrm{msec}$. Sounds were delivered five times by each loudspeaker, resulting in a total of 95 localization trials. Presentation order of loudspeakers was irregular, but balanced for frequency of occurrence. A train of stimuli continued until listeners made a location judgment.

When the white noise localization task had been completed, the pretraining session began. Two different acoustic stimuli, each $5.0 \mathrm{kHz}$ in width, were employed. One, referred to as the middlefrequency noise band, was centered at $6.0 \mathrm{kHz}$; the other, referred to as the high-frequency noise band, was centered at $12.0 \mathrm{kHz}$.
Hence, the middle-frequency and high-frequency noise bands extended from 3.5 to $8.5 \mathrm{kHz}$ and from 9.5 to $14.5 \mathrm{kHz}$, respectively. Both bands were fabricated through the use of a ring modulator system by which a carrier (center) frequency was modulated by a 2.5-kHz low-pass noise. This produced a bandpass noise symmetrical on each side of the center frequency with a slope of approximately $10 \mathrm{~dB}$ per $400 \mathrm{~Hz}$. Stimulus duration, rise-fall time, repetition rate, and sensation levels remained the same as those described for the white noise localization session. Listeners were asked to localize, monaurally, both frequency bands; they were given 95 trials on each.

Upon completion of the pretraining session, the subjects were randomly assigned to two groups of 6 for the training sessions. One group was trained to locate the middle-frequency band, and the other was trained to locate the high-frequency band. Training consisted of feedback immediately following the listener's response to the stimulus train. For example, a band of noise would be delivered by Loudspeaker No. 4 , which was placed $30^{\circ}$ left of the midline. If the listener responded by calling out "Four," the experimenter, through an intercom, would say "Correct" and would repeat the stimulus train. If the listener called out a different number, the experimenter would announce the number of the correct loudspeaker, in this case No. 4, and would repeat the train of noise bursts. Following a few seconds of silence, the experimenter would begin the next trial. The listeners were trained for five sessions over a 2 -week period, with each session consisting of 95 trials. Irrespective of its frequency content, the noise band on which listeners were trained to localize is referred to as the experimental stimulus. The other noise band (the control stimulus) was not presented during the training sessions. 1

Within 3 days after the final training session, Posttest Session 1 was given. In this session, listeners were asked to locate both the experimental and control stimuli. Feedback on location judgments was withheld. Posttest Session 2 was given 2 to $21 / 2$ months later. Location judgments on the experimental and control stimuli were recorded; feedback was again withheld.

Performance was evaluated in terms of error score. An error score of $\mathbf{0}$ was entered if the listener chose the loudspeaker delivering the sounds. An error score of $1,2,3, \ldots$ was entered if the listener chose as the sound source the loudspeaker that was $1,2,3, \ldots$ loudspeakers removed from the correct one.

\section{Results}

Figure 1 illustrates the phenomenon that served as the impetus for this investigation: Unpracticed listeners, when locating a sound monaurally, will displace it away from the midline and toward the unoccluded ear. Note that variability in location judgments also increases for sounds emanating from the vicinity of midline. Figure 2 summarizes, in graphic form, the location errors for the experimental and control stimuli. Mean location errors (in degrees) are plotted for the various test sessions. The listeners performed poorly when locating the middle- and high-frequency noise bands during the pretraining session; however, performance improved dramatically once feedback was provided. A two-way repeated measures analysis of variance was carried out on mean error scores recorded for the sequence of sessions beginning with the pretraining session and ending with Posttraining Session 1. Mean error scores were analyzed as a split plot wherein listeners were nested within frequency and completely crossed with sessions. The main effect of sessions was significant $[F(6,60)=33.5, p<.001]$. On the other hand, the main effect of frequency content (middle band 


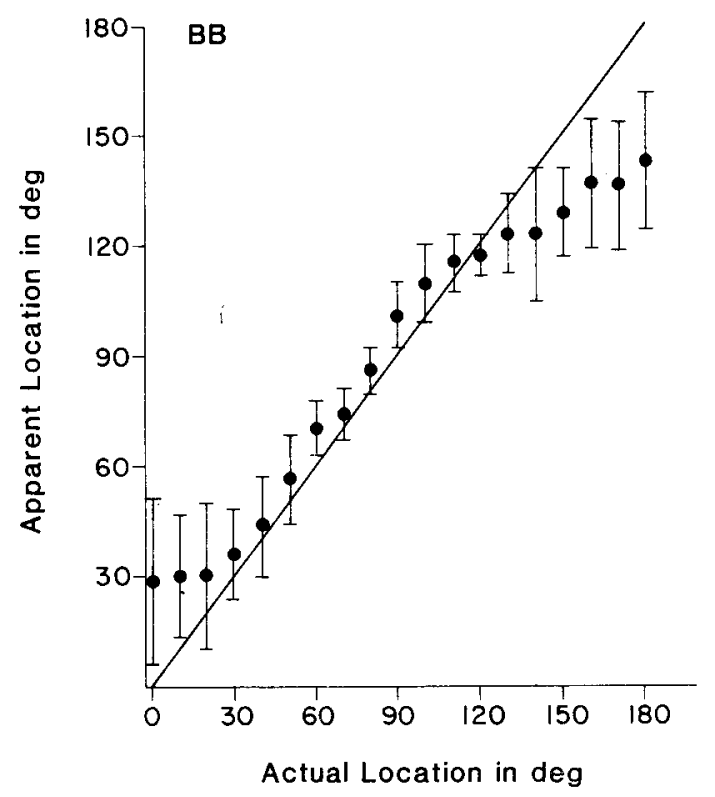

Figure 1. Mean apparent location of a broadband (BB) noise as a function of its actual location. Lengths of vertical lines indicate \pm 1 standard deviation.

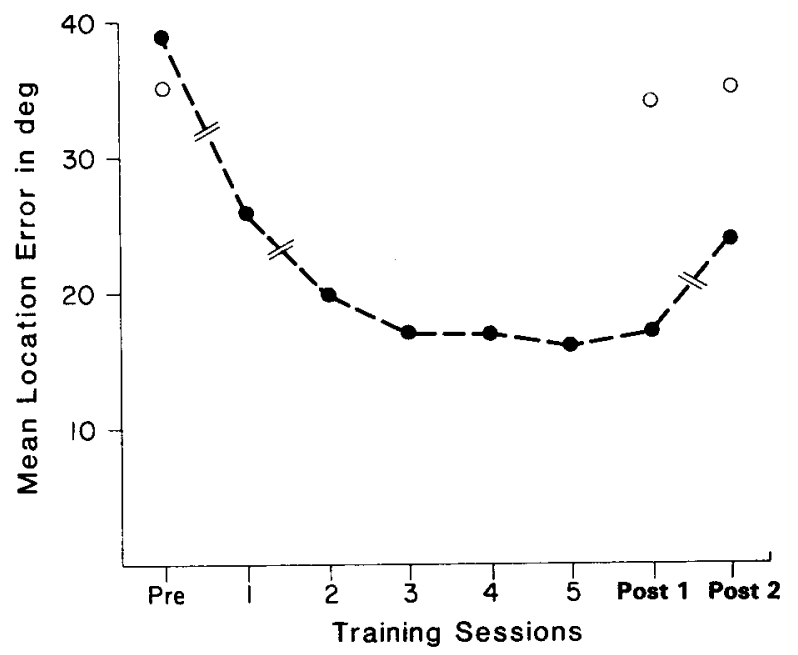

Figure 2. Mean location errors for the experimental stimulus (closed circles) and the control stimulus (open circles).

vs. high band) failed to reach significance $[F(1,10)=.46$, $p>.05]$, thereby suggesting that both noise bands profited equally from training. Tukey's honestly significant difference (HSD) test indicated that the mean location error score for the pretraining session differed from those of all other sessions $(p<.05)$ and that the mean location error score for Day 1 of the training session differed from those of Day 2 and subsequent training sessions $(p<.05)$. The mean error score for localization of the experimental stimulus declined on Posttraining Session 2 vis-à-vis Posttraining Session 1 ( $p<.05 ; t$ test), but remained greater than that obtained on the pretrain- ing session. For localization of the control stimulus, performance was poor on the original test session (mean location error $=35.2^{\circ}$ ) and showed no sign of improvement on subsequent tests (see Figure 2). In short, training to locate sounds characterized by one set of spectra did not affect location performance on sounds characterized by a different set.

Another view of the data is presented in Figure 3, which illustrates where the improvement in localization performance occurred as the result of practice. Although displacement from the midline was still evident in Posttraining Session 1, its extent was greatly reduced. Upon retesting about 2 months later, however, listeners displayed an increased tendency to displace the sounds away from the midline (see Post- $T_{2}$ symbols). Location judgments for sounds originating from $30^{\circ}$ to $120^{\circ}$ left of midline, however, maintained their accuracy on Posttraining Session 2.

\section{EXPERIMENT 2}

\section{Association Between Head-Related Transfer Functions and Location Judgments}

\section{Method}

Our method for measuring the transfer functions for acoustic stimuli has been published (Musicant \& Butler, 1984). Briefly, the subject was seated in the room where the localization tests were conducted. A Knowles miniature microphone (Model BT 1759) was fitted in the left external canal and held firmly in place by a pliable plastic compound. The listener was informed that we would record from the microphone and that it was of utmost importance that the subject keep his/her head firmly against the headrest. The participants were those tested in the first experiment. Measurements on each were carried out individually. Noise bands, $1.0 \mathrm{kHz}$ wide and centered at 4.0 through $14.0 \mathrm{kHz}$ in steps of $1.0 \mathrm{kHz}$, were

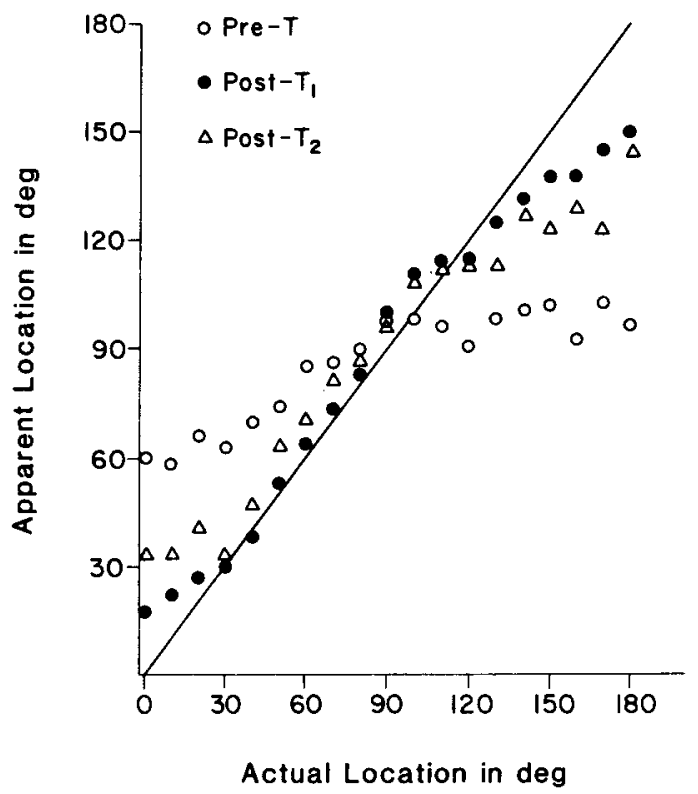

Figure 3. Mean apparent location of the experimental stimulus as a function of its actual location. Only the data for pretraining (Pre-T) and posttraining (Post-T and Post- $T_{2}$ ) sessions are presented. 
presented singly from each loudspeaker. With the intensity of the acoustic input fixed, the microphone's output was amplified and fed to a vacuum tube voltmeter, and the resulting value was tabulated. A session consisted of 209 measurements: 11 differently centered noise bands presented from 19 loudspeaker locations. The entire procedure was repeated on another day. On four occasions, which were interspersed with the pinna recordings, a complete set of measurements was made with the microphone positioned in the space normally occupied by the subjects' head. The means of these measurements served as the reference for calculating the relative amplification provided primarily by each subject's pinna for each noise band generated by each loudspeaker.

To augment our data base on the association between HRTFs and location judgments, an additional set of tests was carried out. The subjects were requested to locate the narrow-band noise bands used to generate the HRTFs. The temporal characteristics of the stimuli were the same as those employed in Experiment 1. Free-field thresholds for the 1.0-kHz-wide noise bands were obtained for those bands centered at 4.0, 7.0,11.0, and $14.0 \mathrm{kHz}$. Thresholds for the remaining noise bands were estimated via interpolation. During the location tests, the narrow-band noise bursts were presented at approximately $30 \mathrm{~dB}$ SL. Unbeknown to the listeners, all noise bursts were generated by the loudspeaker positioned $90^{\circ}$ left of the midline. During a test session, each differently centered noise burst was presented five times in an irregular order. Location judgments were collected over four sessions, resulting in a total of 20 judgments for each of the 11 different noise bursts.

\section{Results}

The peaks of each listener's transfer functions for the differently centered noise bands were extracted from the data and the frequency distributions of these peaks were plotted against their center frequencies (Figure 4). The circled areas in the figure represent the number of subjects whose HRTFs, generated by a given noise band, peaked at the same location. The largest circle signifies

\section{Location of Peak Amplification (deg)}

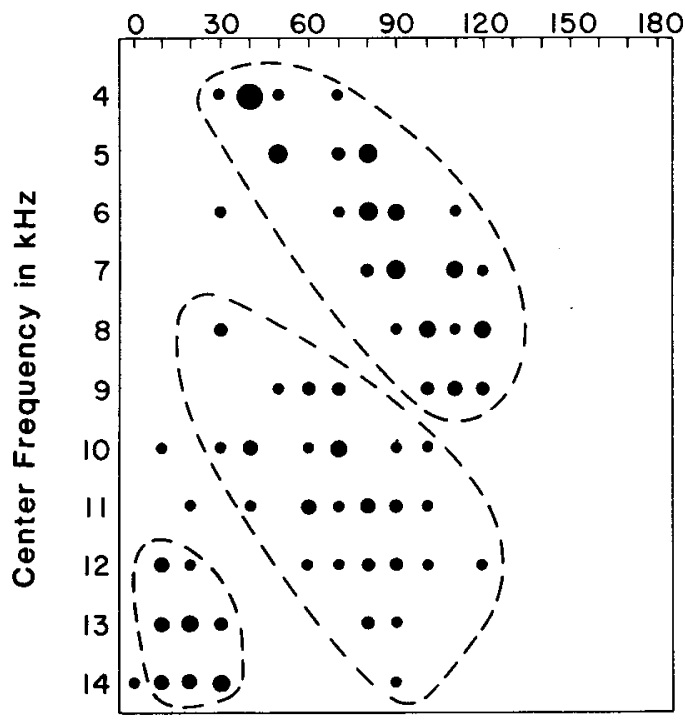

Figure 4. Location of peak amplification as a function of center frequency. Circled areas represent frequency of occurrence as described in text. that 9 listeners' functions peaked at the same azimuthal position; the smallest denotes a location at which only 1 listener's function peaked. By and large, the HRTFs peaked in response to noise bands presented from $40^{\circ}$ to $120^{\circ}$ left of the midline. Only for noise bands centered at 12.0 through $14.0 \mathrm{kHz}$ did the function peak at nearmidline locations. Regardless of the noise bands' frequency content, no HRTF function peaked at locations beyond $120^{\circ}$.

Location judgments of the narrow noise bands were linked to the bands' center frequencies in much the same way as reported previously (Butler \& Flannery, 1980). The apparent location of the narrow bands moved toward the side as the center frequency was increased from 4.0 to about $8.0 \mathrm{kHz}$, then switched to the frontal region when the center frequency was 8.0 or $9.0 \mathrm{kHz}$, only to migrate again toward the side with further increments in center frequency. At the highest center frequencies, most listeners perceived the sounds to originate once more in the frontal region. The pinna data in Figure 4 were circled to draw attention to the parallel between the pattern of functions' peaks and that of location judgments.

Using the mean free-field threshold for the 4.0-kHzcentered noise band as a reference, mean thresholds for the 7.0-, 11.0-, and 14.0-kHz-centered bands were elevated by $3.7,5.1$, and $11.7 \mathrm{~dB}$, respectively. These data have direct relevance to an issue addressed in the Discussion section.

To provide a more detailed picture of the association between HRTFs and location judgments of those narrow bands that generated the functions, the data of two listeners, M.G. and O.G., are presented in Figures 5 and 6 . The numbers directly above the points on the functions represent the percentage of total presentations of each center frequency on which the various azimuthal positions were judged as the origin of the sounds. As mentioned earlier, each center frequency was presented 20 times in an irregular order. The judged locations tended to cluster around the peak of the function generated by each noise band, despite the fact that all stimuli during the localization sessions originated from $90^{\circ}$ left of the midline. Listener O.G.'s location responses, however, did not coincide as closely with the peak of the functions generated by noise bands centered at 13.0 and $14.0 \mathrm{kHz}$ (Figure 6). Some of the other listeners also showed a lack of correspondence between location judgments and peaks of the functions at the highest center frequencies. The pretraining localization performances of M.G. and O.G. on the middle- and high-frequency noise bands are shown in Figure 7. Notice that for Listener M.G., location judgments for the middle-frequency noise bands extended farther toward the rear than did his location judgments for the high-frequency noise bands. His HRTFs for frequencies composing the middle-frequency noise band also peaked progressively farther toward the rear, as did his location judgments for the same frequencies (Figure 5). Listener O.G.'s localization data and his HRTFs followed a similar pattern (Figures 6 and 7). 


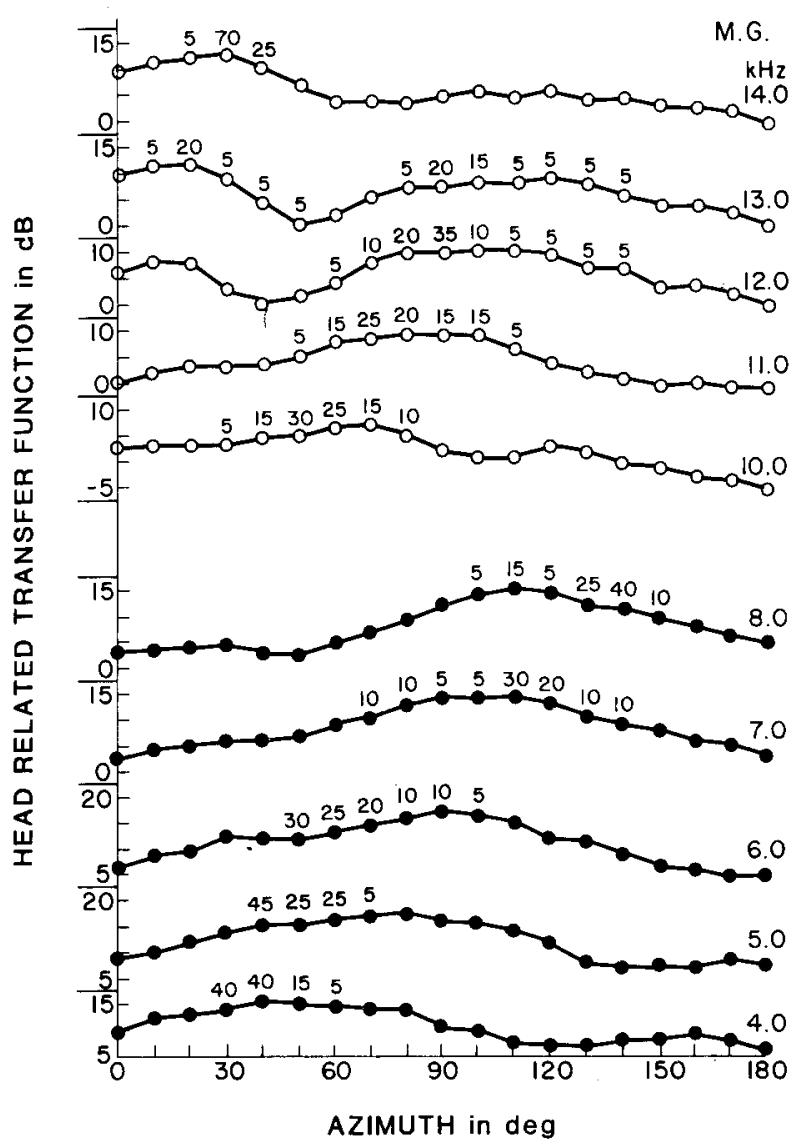

Figure 5. Listener M.G.'s head-related transfer function generated by narrow noise bands differing in center frequency. See text for meaning of numbers arrayed above each curve.

Taken together, these correspondences between location judgments of middle- and high-frequency noise bands and HRTFs lend credence to the claim that spectral peaks serve as a guide for monaural localization. Consistent with the report of Musicant and Butler (1984), the location judgments for the narrow noise bands distributed themselves around the crests of the transfer functions, thereby demonstrating an association between the two sets of data. The range of location judgments of the middle- and highfrequency noise bands could have been estimated reasonably well either by the positions of the spectral peaks or by the location judgments of the 1.0-kHz-wide frequency segments encompassed by the wider bands. Particularly germane to the purpose of the present study is that spectral peaks did not occur at or near midline positions; with rare exceptions, neither did localization responses.

\section{DISCUSSION}

This study was motivated by the idea that monaural displacement of sounds away from the midline and toward the functioning ear may be due to the absence of a natural cue for locating sounds originating on or near the $0^{\circ}-180^{\circ}$ axis; that this cue is the covert peak in the HRTF; that monaural training that enables a listener to localize accurately sounds emanating from the midline is a consequence of acquiring a highly specific set of spectral cues; and that such training will not benefit performance in locating sound of a different spectral composition. The question of whether the data can adequately be explained within this conceptual framework is addressed in the following paragraphs.

The perceptual phenomenon was first recreated by requesting naive listeners to localize broadband noise. Indeed, those sounds originating at or near the midline were displaced toward the functioning ear. So, too, were the middle-frequency and high-frequency noise bands when listeners judged their location during the pretraining session: mean location judgments ranged from about $60^{\circ}$ to approximately $105^{\circ}$ for sounds originating from $0^{\circ}$ to $180^{\circ}$ left of midline. None of the HRTFs peaked at azimuthal locations beyond $120^{\circ}$. Thus, the behavioral data and microphone measurements for sounds coming from the rear are in accordance with the proposition that a covert peak in the transfer function serves as a natural

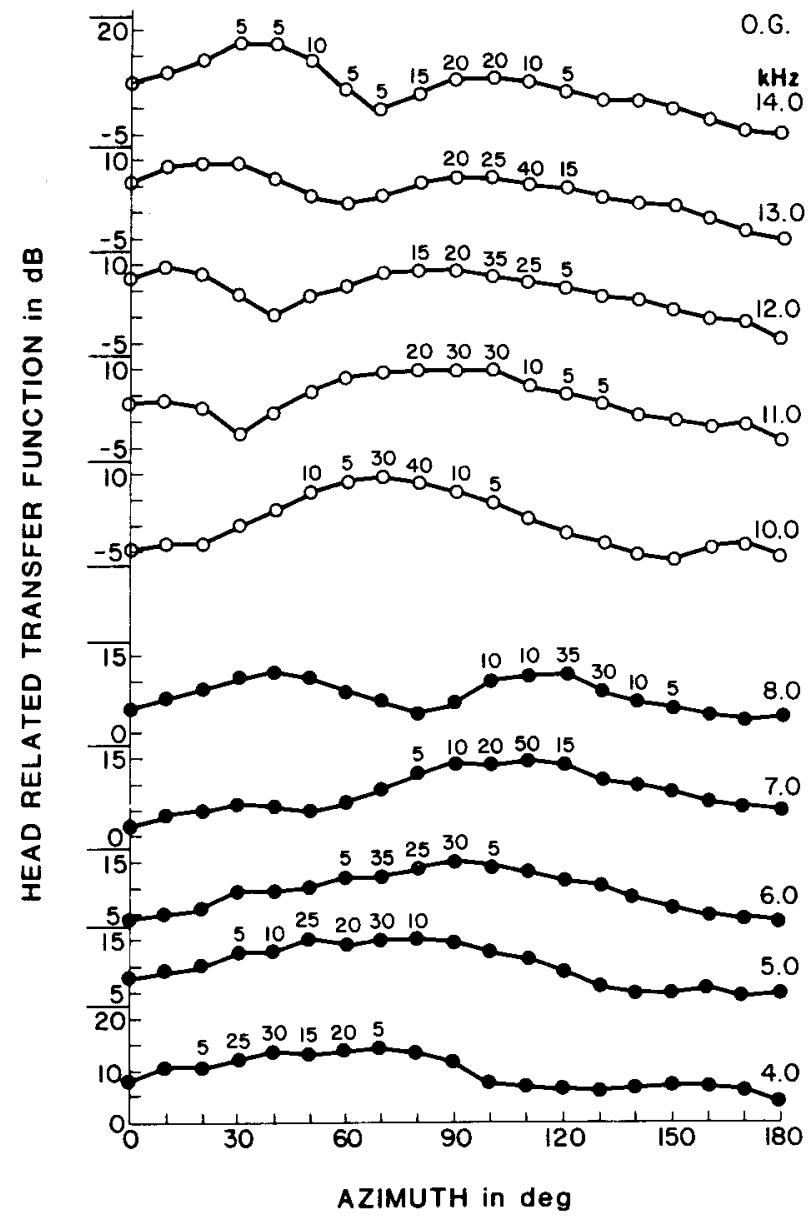

Figure 6. Listener O.G.'s head-related transfer function generated by marrow noise bands differing in center frequency. See text for further description. 


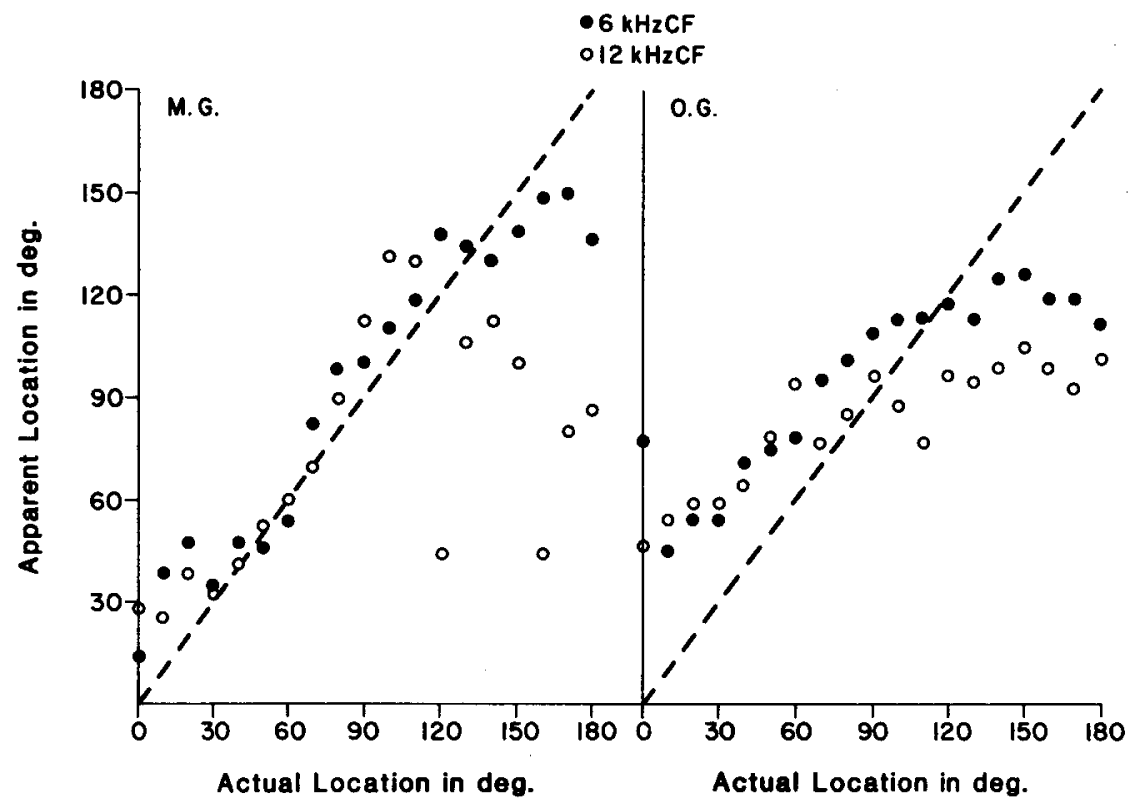

Figure 7. Mean apparent location of the middle- and high-frequency bands as a function of their actual location. Listener M.G.'s performance data are shown on the left, those of Listener O.G. on the right.

cue for location, and in its absence the location of the sound is perceived to be elsewhere. For sounds coming from the front, with few exceptions, the transfer function did not peak at frontal locations $\left(\leq 30^{\circ}\right)$ for those narrow noise bands whose frequency content was encompassed by the middle-frequency noise bands (3.5-8.5 kHz); accordingly, location judgments for this band were displaced toward the side. But so were judgments of the high-frequency band. In the latter case, transfer functions generated by the 1.0-kHz-wide noise bands centered at 13.0 and $14.0 \mathrm{kHz}$ did peak when these stimuli originated from near the front $\left(10^{\circ}\right.$ to $30^{\circ}$ left of midline). Perhaps this discrepancy can be accounted for in terms of a reduction in threshold for frequencies as high as 13.0 and $14.0 \mathrm{kHz}$. As reported in Results of Experiment 2, group mean thresholds for the 1.0-kHz-wide noise band centered at $14.0 \mathrm{kHz}$ were elevated by approximately $7 \mathrm{~dB}$ over those calculated for the $11.0-\mathrm{kHz}$ centered narrow noise band. Hence, when the highfrequency band $(9.5-14.5 \mathrm{kHz})$ was presented at $30 \mathrm{~dB}$ SL, the 13.0-14.0-kHz region was attenuated relative to the lower frequencies within the band, and its influence on location judgments was reduced accordingly.

The most noticeable effect of training was a decrease in the displacement of sounds from the midline as attested to by the results of Posttraining Session 1. Localization performance improved for the sound on which listeners were trained, that is, the experimental stimulus. No improvement was observed when listeners located the control stimulus. The failure of training on one stimulus to transfer positively to another suggests that spectral cues specific to the experimental stimulus were acquired. One can infer that the learned cues primarily informed listeners about midline-positioned sounds: on Posttraining Session 2 a statistically significant decrease in accuracy was observed when subjects located the experimental stimulus, and this decrease can be attributed principally to the listeners' once more displacing sounds coming from the midline regions (see Figure 3). Much of the beneficial effect of training, however, was retained.

I am cognizant of other interpretations of the training effect, perhaps the simplest being that training informs the listener about the front and rear anchoring points of the space occupied by the loudspeakers. Once these are known, the location judgments of all intermediate positions can be adjusted with regard to the degree of spectral similarities between sounds coming from adjacent loudspeaker locations. This view of the data is not encumbered by the concept of a natural cue for location, but if the concept advances our understanding of monaural localization, it should be employed as a working hypothesis for further investigation. How, then, may the idea of natural cues (covert peaks) enter into the process of localization of a wide-band noise? First, listeners exhibit a strong tendency to localize a narrow noise band at or near the azimuthal position at which this noise band is most amplified, that is, the position of its covert peak. ${ }^{2}$ As proposed at the beginning of this paper, the auditory system somehow detects that frequency segment that is amplified more at a particular azimuthal position than at any other position; that is, it detects the covert peak. Since every frequency segment encompassed by a wide-band noise possesses a spatial referent (Butler \& Flannery, 1980), the spatial referent for the frequency segment that 
peaks covertly when the noise comes from a certain position in the horizontal plane informs the listener about the location of the noise. If a wide-band noise originates at an azimuthal position at which none of its inherent frequency components generates a covert peak, the sound's apparent location will be displaced toward the functioning ear, as it was in the initial (white noise) test in the present study. The relatively large variability of location judgments of the white noise emanating at or near the midline (Figure 1) indicates that untrained listeners apparently have no simple, unequivocal cue for monaurally locating sounds coming from the front or rear.

These data weaken the binaural imbalance concept as an explanation for monaural displacement from the midline, because interaural intensity differences that result from occluding one ear cannot account for the pattern of perceived displacement of the differently centered narrow bands of noise. Some frequency segments are greatly displaced, others less so. On the other hand, it may be the overall shape of the spectrum that serves as the cue for localization, with spectral peaks being merely one of the characteristics of the pattern. This idea is supported by data that show an orderly improvement in monaural localization with increases in bandwidth (Butler, 1986). The spectral pattern becomes progressively elaborated, thereby furnishing a wealth of cues. It should be mentioned, however, that as bandwidth is increased, more than one frequency segment within the band peaks covertly when the sound emanates from a given position. Perhaps it is this redundancy in covert spectral peaks, and not a concatenation of additional cues, that contributes to improved localization with bandwidth enhancement.

Finally, if it is indeed the covert peaks of the transfer functions that provide a natural cue for monaural localization, one can understand why listeners may not reach a high level of performance even when sounds originate from the side-the peaks are not sharp. Hence, practice benefits performance in locating sound from the middle sector of the horizontal plane, as well as that from the front and rear sectors.

\section{REFERENCES}

ANSI (1969). American national standard specification for audiometers (ANSI S3.36-1969). New York: American National Standards Institute.

BUTLER, R. A. (1986). The bandwidth effect on monaural and binaural localization. Hearing Research, 21, 67-73.

BUtler, R. A., \& FlanNery, R. (1980). The spatial attributes of stimulus frequency and their role in monaural localization of sound in the horizontal plane. Perception \& Psychophysics, 28, 449-457.

Musicant, A. D., \& Butler, R. A. (1980). Monaural localization: An analysis of practice effects. Perception \& Psychophysics, 28, 236-240.

Musicant, A. D., \&utler, R. A. (1984). The psychophysical basis of monaural localization. Hearing Research, 14, 185-190.

PIERCE, A. H. (1901). Studies in auditory and visual space perception. New York: Longmans, Green.

Shaw, E. A. G. (1974). The external ear. In W. D. Keidel \& W. D. Neff (Eds.), Handbook of sensory physiology: Vol. 5, Pt. 1. Auditory System. Berlin: Springer-Verlag.

Stevens, S. S., \& Newman, E. B. (1936). The localization of actual sources of sound. American Journal of Psychology, 48, 297-306.

\section{NOTES}

1. This part of the study was concerned with the effects of training to localize sounds monaurally versus no training, not the effects of feedback versus no feedback.

2. That location judgments and covert peaks did not correspond more closely was not unexpected. Listeners' location judgments can be influenced by cognitive as well as stimulus factors. Listeners develop their own strategies for coping with a difficult localization task, strategies they occasionally reveal to the experimenter after a test session. Furthermore, the sound spectrum measured at the entrance of the ear canal is not that which is expressed at the tympanic membrane. The discrepancy could adversely affect the correlation between the HRTF covert peaks measured in this study for the variously centered $1.0-\mathrm{kHz}$-wide noise bands and the apparent location of these noise bands.

(Manuscript received June 20, 1986; revision accepted for publication November 10, 1986.) 